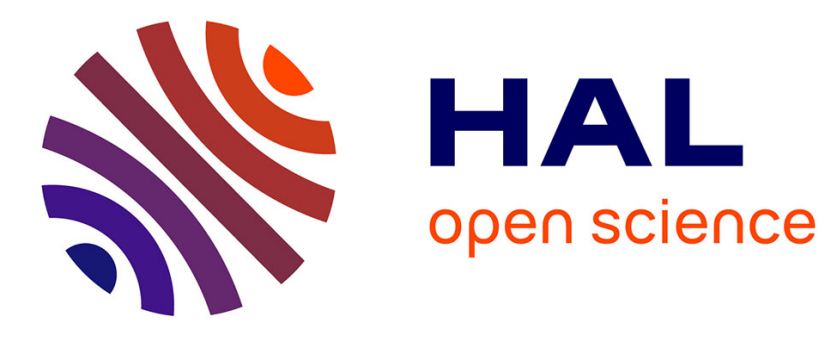

\title{
Molecular beam epitaxial growth of hexagonal boron nitride on $\mathrm{Ni}$ foils
}

Jawad Hadid, Ivy Colambo, Christophe Boyaval, Nicolas Nuns, Pavel Dudin, Jose Avila, X. Wallart, Dominique Vignaud

\section{- To cite this version:}

Jawad Hadid, Ivy Colambo, Christophe Boyaval, Nicolas Nuns, Pavel Dudin, et al.. Molecular beam epitaxial growth of hexagonal boron nitride on Ni foils. 2D Materials, 2021, 10.1088/20531583/ac1502 . hal-03292918

\section{HAL Id: hal-03292918 \\ https://hal.science/hal-03292918}

Submitted on 20 Jul 2021

HAL is a multi-disciplinary open access archive for the deposit and dissemination of scientific research documents, whether they are published or not. The documents may come from teaching and research institutions in France or abroad, or from public or private research centers.
L'archive ouverte pluridisciplinaire HAL, est destinée au dépôt et à la diffusion de documents scientifiques de niveau recherche, publiés ou non, émanant des établissements d'enseignement et de recherche français ou étrangers, des laboratoires publics ou privés. 


\title{
Molecular beam epitaxial growth of hexagonal boron nitride on Ni foils
}

\author{
Jawad Hadid ${ }^{1}$, Ivy Colambo ${ }^{2}$, Christophe Boyaval ${ }^{1}$, Nicolas Nuns ${ }^{3}$, Pavel Dudin ${ }^{4}$, Jose Avila ${ }^{4}$, Xavier \\ Wallart $^{1}$, Dominique Vignaud ${ }^{1 *}$ \\ ${ }^{1}$ Univ. Lille, CNRS, Centrale Lille, JUNIA ISEN, Univ. Polytechnique Hauts de France, UMR 8520-IEMN F- \\ 59000 Lille France \\ ${ }^{2}$ Inst. Math. Sci. Phys., Univ. of the Philippines Los Banos, Laguna 4031 Philippines \\ ${ }^{3}$ Univ. Lille, CNRS, Centrale Lille, ENSCL, Univ. d'Artois, IMEC-Institut Michel-Eugène Chevreul 59000 \\ Lille France \\ ${ }^{4}$ Synchrotron SOLEIL \& Université Paris-Saclay,F-91192 Gif sur Yvette France \\ *corresponding author: dominique.vignaud@univ-lille.fr
}

\begin{abstract}
:
Hexagonal boron nitride was synthesized by molecular beam epitaxy on polycrystalline $\mathrm{Ni}$ foils using borazine $\left(\mathrm{B}_{3} \mathrm{~N}_{3} \mathrm{H}_{6}\right)$ as precursor. Our photoemission analysis shows that several components of boron and nitrogen are detected, suggesting the complex nature of the bonds noticeably at the $\mathrm{h}-\mathrm{BN} / \mathrm{Ni}$ interface. The BN thickness was estimated by photoemission and the BN distribution by ToF-SIMS. Due to the catalytic effect of the Ni substrate, this thickness is self-limited in the range 1-2 layers regardless of the borazine dose. A spatially resolved photoemission study was carried out before and after transfer of the h-BN on a Si substrate. It shows that a strong electronic coupling exists at the interface between h-BN and polycrystalline $\mathrm{Ni}$, not only for (111) grains, which disappears after transfer on Si. In addition, we highlight the importance of detecting $\pi$ plasmons in the photoemission spectra to confirm the hexagonal nature of boron nitride.
\end{abstract}




\section{Introduction:}

Graphene exhibits several attractive material and electrical properties. However, due to its atomically thin 2D nature, these properties strongly depend on the materials which are in close contact with it, regardless of whether they are a substrate, a gate dielectric or else. This implies that any interaction with its surroundings may negatively affect its electronic properties. Recently, numerous studies have shown that hexagonal boron nitride (h-BN) can be considered as a suitable substrate to preserve the exceptional properties of the graphene. With a lattice mismatch of 1.6\% [1], van der Waals inter-planar bonds [2], a wide band gap of $6.0 \mathrm{eV}[3-4], \mathrm{h}-\mathrm{BN}$ has proven to be an ideal layer for encapsulating graphene [5].

Despite the progress and the diversity of growth techniques, current research still faces the challenge of synthesizing BN with large dimensions while controlling the number of layers. Compared to graphene where growth can occur by a purely surface process [6-7], the growth mechanisms in the case of h-BN are intrinsically more complex, because two elements are involved and must be incorporated into the $B N$ film at a 1:1 ratio. Although the stoichiometry should be ensured by the use of molecules such as borazine where the B: $N$ ratio is 1 , the decomposition of the precursor and the pressure play an important role in improving the crystalline quality of $B N$. The thermal decomposition of borazine $\left(\mathrm{B}_{3} \mathrm{~N}_{3} \mathrm{H}_{6}\right)$ on several substrates generates a chemisorbed or physisorbed self-limited BN layer [8-10]. The metal-BN interactions depends on the substrate, the electronic coupling at the interface ranging from very low on $\mathrm{Pt}$ to high on $\mathrm{Ni}$. In the case of $\mathrm{Ni}(111)$, chemisorption results in a much higher density of states at the interface than on Pt(111), the h-BN monolayer on Ni being metallic [8]. On Rh(111), the nanomesh structure [11] also results in electronic states at the interface, but with a lower density than on $\mathrm{Ni}(111)$. On the other hand, it seems that one- or two-plane self-limitation of the h-BN thickness is mostly encountered at low-pressure growth conditions. This hypothesis is in agreement with the results of Nagashima et al. [9] and Preobrajensky et al. [10], which showed that in the case of $\mathrm{Ni}(111)$, the formation of the second layer of h-BN requires a much higher dose of borazine than that needed to form the first plane.

In this article, we study the growth of h-BN by molecular beam epitaxy (MBE) on polycrystalline $\mathrm{Ni}$ foils using borazine $\left(\mathrm{B}_{3} \mathrm{~N}_{3} \mathrm{H}_{6}\right)$ as precursor [8-10]. Because this molecule is used in a gaseous state in an MBE chamber, this technique is best described as gas source MBE. Here, we combine conventional X-ray photoemision spectroscopy (XPS), spatially resolved XPS and time-of-flight secondary ion mass spectroscopy (TOF-SIMS) characterizations. We clearly demonstrate that BN is deposited on the whole surface with a thickness varying between 1 and $2 \mathrm{MLs}$, the average film thickness being limited by the catalytic effect of the Ni substrate. We evidence the strong Ni substrate/BN coupling whose characteristic features disappear after transfer on a Si substrate. These results extend those reported on the $\mathrm{Ni}(111)$ surface [8-10] to polycrystalline foils. Finally, we underline the importance of detecting $\pi$ plasmons in photoemission spectra to confirm the $2 \mathrm{D}$ nature of $\mathrm{h}$ BN.

\section{Experimental methods:}

All growth experiments were carried out in a commercial RIBER MBE chamber under UHV conditions (base pressure $\sim 8 \times 10^{-11}$ Torr). The growth temperature was set between 700 and 
$1050^{\circ} \mathrm{C}$, measured by an infrared pyrometer (at a wavelength of $0.85 \mu \mathrm{m}$ ). The borazine was purchased from the Katchem company, its purity being specified to be higher than $97 \%$. It is stored in a stainless steel bottle at low temperature $\left(-15^{\circ} \mathrm{C}\right)$. To increase the partial pressure of borazine relative to any residual gases, its temperature is set to $10^{\circ} \mathrm{C}$ during growth. The borazine flux is controlled by a regulating pressure system positioned between the borazine bottle and a calibrated $0.25 \mathrm{~mm}$ diameter hole, and enters the MBE chamber through a gas injector set at $300^{\circ} \mathrm{C}$. Borazine vapor is not expected to be fragmented at this temperature [12]. For a regulation set at 0.1 or 1 Torr respectively, the pressure in the growth chamber is $3.5 \times 10^{-8}$ or $4.5 \times 10^{-7}$ Torr. The $25 \mu \mathrm{m}$ thick polycrystalline Ni foils were purchased from AlfaAesar. They were cut in $1 \times 1 \mathrm{~cm}^{2}$ pieces, then ultrasonically cleaned at room temperature for 20 minutes in acetone and in isopropanol and finally deoxidized at $\sim 50^{\circ} \mathrm{C}$ in acetic acid for about 15 minutes. The substrates were systematically annealed at $1000^{\circ} \mathrm{C}$ in the $\mathrm{MBE}$ chamber for at least 1 hour before growth. This annealing step aims to eliminate some of the carbonaceous species adsorbed on the Ni substrates [13], to remove the residual oxygen and to enlarge the Ni grains [14]. It results in grains of typical size between 5 and $50 \mu \mathrm{m}$, without any preferential orientation (see the electron backscattered diffraction image S1 in the supplementary material). To transfer the BN film onto a Si substrate, we relied on a wet etching of the $\mathrm{Ni}$ substrate via a solution of $\mathrm{FeCl}_{3}$. This process was initially developed to transfer graphene [15], it is detailed in the supplementary material.

Conventional and spatially resolved photoemission measurements, Raman spectroscopy, ToF-SIMS and SEM observations were carried out. Raman measurements were done using a Horiba Scientific LabRAM HR confocal spectrometer fitted with a $473 \mathrm{~nm}$ laser (spot size 1 $\mu \mathrm{m}$ and power $\sim 10 \mathrm{~mW}$ ). All spectra presented in this work were corrected by substraction of the Ni substrate contribution. A Zeiss Supra 55 scanning electron microscope was used at an energy of $1 \mathrm{keV}$. Everhart-Thornley and InLens detectors were used. The Everhart-Thornley detector is tilted relative to the electron beam to be more sensitive to secondary electrons than to backscattered electrons. Its image contains information related to the topography of the substrate. The InLens detector mainly measures electrons backscattered along the beam direction and produces images with high spatial resolution. In the case of BN, the InLens intensity depends on the thickness of the deposited material [16]. ToF-SIMS measurements were realized with a TOF SIMS.5 system (IONTOF). Measurements were performed in burst mode for mapping and bunch mode for spectroscopy, using $25 \mathrm{keV} \mathrm{Bi}^{+}$as primary ion source. The typical spatial resolution is $200 \mathrm{~nm}$ for mapping. The BN content was studied by measuring the ${ }^{10} \mathrm{BN}^{-}$signal [17]. Conventional XPS measurements were realized using a Physical Electronics 5600 spectrometer fitted in an UHV chamber (base pressure $\sim 1 \cdot 10^{-10}$ Torr). A monochromatized Al anode X-ray source (at $1487 \mathrm{eV}$ ) was used with a typical probe size of $400 \mu \mathrm{m}$ diameter. The detection angle was varied between $25^{\circ}$ and $75^{\circ}$ with respect to the sample surface plane. The spectra shown here were all obtained with a $45^{\circ}$ angle. Spatially resolved photoemission was also measured at the Antares beamline of the SOLEIL Synchrotron with a photon energy of $700 \mathrm{eV}$, a photon probe size of $\sim 0.5 \mu \mathrm{m}$ diameter and a detection normal to the surface. At Antares, all samples were first annealed under UHV conditions to eliminate any possible contamination resulting from the air transfer. The annealing temperature was fixed at $700^{\circ} \mathrm{C}$, lower than the growth temperature $\left(800^{\circ} \mathrm{C}\right)$. To optimize the beamtime use, the mappings were limited to the $\mathrm{N} 1 \mathrm{~s}$ core level and the presence of $\pi$ plasmons was only evaluated for the N1s spectrum (nitrogen having a higher sensitivity factor than boron). 


\section{Results:}

\subsection{Catalytic effect of the Ni substrate:}

A typical h-BN XPS spectrum shows a single B1s peak between 190 and $191 \mathrm{eV}$ and a single $\mathrm{N} 1 \mathrm{~s}$ one in the range 397.6-398.7 eV [18-20]. A signal originating from the $\pi$ plasmons should be detected at $\sim 9 \mathrm{eV}$ from B1s and N1s main peaks to confirm the 2D nature of h-BN [21-22]. Note that previous h-BN photoemission studies often present the B1s and N1s peaks with a reduced energy range that does not include the $\pi$ plasmon energy [18-20] and confirm the 2D nature by complementary measurements (e.g. Raman spectroscopy) without focusing on the detection of $\pi$ plasmons. In our case, XPS measurements were recorded covering a wider energy range that includes the $\pi$ plasmon energy. They indicate the presence of one peak for nitrogen (figure 1a) and two well-resolved components for boron (figure 2a), but the $\pi$ plasmons were not detected regardless of the growth conditions (figures $1 \mathrm{a}$ and $\mathrm{S} 2$ in the supplementary material). The origin of the B1s peak at $188.2 \mathrm{eV}$ (figure 2a) is not well understood. It could correspond to B-Ni [23-24] or B-B bonds [24-26]. The disappearance of this peak after transfer on $\mathrm{Si}$ (see section 3.2) indicates that it is an interface component, regardless of its origin $\mathrm{B}-\mathrm{B}$ or $\mathrm{B}-\mathrm{Ni}$. This peak is not related to $\mathrm{BN}$ and its discussion is beyond the scope of this article.

Figures $1 \mathrm{a}$ and $2 \mathrm{a}$ are high-resolution core-level $\mathrm{B} 1 \mathrm{~s}$ and $\mathrm{N} 1 \mathrm{~s}$ spectra of h-BN on Ni respectively. The best fit for the $\mathrm{B} 1 \mathrm{~s}$ and N1s main peaks involves one single asymmetric component, using a Shirley baseline. The B: $\mathrm{N}$ ratio deduced from the intensity of these two asymmetric peaks is 1.04 and corresponds to the expected stoichiometry of the h-BN within experimental errors. The asymmetric shape of the peaks implies that the synthesized BN has a metallic character, indicating a strong interaction between $\mathrm{BN}$ and the nickel substrate. This metallic nature, already proposed by several articles in the case of h-BN on $\mathrm{Ni}[8,27]$, is consistent with the non-detection of $\pi$ plasmons characteristic of this $2 \mathrm{D}$ material.

The amount of BN deposited on Ni foils was explored as a function of the dose of borazine and of the growth temperature. For this, a model based on a uniform BN film on $\mathrm{Ni}$ (see supplementary material) was used to calculate the $d / \lambda_{B, B N}$ ratio (figure 3 a), where $d$ is the BN thickness and $\lambda_{B, B N}$ is the inelastic mean free path of photoelectrons emitted from $B$ and passing through $\mathrm{BN}$. The $\mathrm{B}: \mathrm{N}$ composition ratio is also plotted in figure $3 \mathrm{~b}$. Above $800^{\circ} \mathrm{C}$, the amount of $\mathrm{N}$ in the deposited layer is reduced and even vanishes when the growth temperature exceeds $900^{\circ} \mathrm{C}$, suggesting that at least $N$ is sublimating at these high temperatures. The optimum growth temperature of $\mathrm{BN}$ on Ni using borazine is thus $800^{\circ} \mathrm{C}$. It is also remarkable that during these isochronous experiments, the $d / \lambda_{B, B N}$ ratio always stands in the range $0.17 \pm 0.02$ between $700^{\circ} \mathrm{C}$ and $800^{\circ} \mathrm{C}$, whatever the pressure of borazine is between $3.5 \times 10^{-8}$ and $4.5 \times 10^{-7}$ Torr. Considering a value of $3.3 \mathrm{~nm}$ for $\lambda_{B, B N}$ for conventional XPS experiments [28], the measured $d / \lambda_{B, B N}$ ratio corresponds to a BN thickness of $\sim 1.7 \mathrm{ML}$ (monolayer).

ToF-SIMS measurements on a BN on Ni sample grown at $800^{\circ} \mathrm{C}$ shows that the ${ }^{10} \mathrm{BN}^{-}$signal is detected everywhere on the $500 \times 500 \mu \mathrm{m}^{2}$ surface (figure 4). This shows that the BN layer is quasi-uniform on the scale of the conventional XPS measurements (probed size is $\sim 400 \mu \mathrm{m}$ ) and hence justifies the $d / \lambda_{B, B N}$ calculation model used (see supplementary material). The fact that the thickness saturates to $\sim 1.7 \mathrm{ML}$ regardless of the growth parameters (dose of 
borazine and growth temperature), shows the critical role of the catalytic effect of the nickel substrate in the BN growth mechanisms using borazine [8-10]. Once a BN layer is formed, the direct $\mathrm{Ni}$-borazine interaction disappears, the substrate loses its impact to break down the molecules and the growth is stopped. This self-limitation is also related to the very low solid solubility of $\mathrm{N}$ in $\mathrm{Ni}$ [29], which prevents the migration of $\mathrm{N}$ towards the Ni bulk during the initial growth phase followed by out-diffusion towards the interface during the cooling phase. This last set of processes were found to be active in the case of graphene growth on $\mathrm{Ni}$, resulting in thick graphene layers [30]. The active growth mechanisms are different for graphene and BN although the same Ni substrate is used.

\section{2 h-BN/Ni interactions:}

The interactions between $\mathrm{BN}$ and the $\mathrm{Ni}$ substrate were studied by ToF-SIMS and photoemission before and after transfer of the BN film. Figure 5 compares the ToF-SIMS spectra measured on the BN sample for which the mapping was recorded (figure 4) and on a reference $\mathrm{Ni}$ foil. In figure $5 \mathrm{a}$, a peak corresponding to ${ }^{10} \mathrm{BN}^{-}$fragments is detected at $\mathrm{m} / \mathrm{z}=$ 24.02. The peak at $m / z=24.00$ corresponding to carbon fragments is more intense on the BN sample than on the nickel reference due to a longer exposure to ambient air. The detection of a peak at 82.95 (figure 5b) may originate from two different fragments, $\mathrm{CHCl}_{2}{ }^{-}$or $\mathrm{BNNi}^{-}$. However, figure $5 \mathrm{c}$ shows that the $\mathrm{Cl}^{-}$peak at $\mathrm{m} / z=34.97$ is $\sim 40 \%$ more intense in the reference $\mathrm{Ni}$ foil than in the $\mathrm{BN}$ sample. This shows that, if the peak at $\mathrm{m} / z=82.95$ only results from $\mathrm{CHCl}_{2}{ }^{-}$ions in the reference $\mathrm{Ni}$ foil, the same peak is dominated by the presence of $\mathrm{BNNi}^{-}$ fragments for the $\mathrm{BN}$ on $\mathrm{Ni}$ sample. The detection of $\mathrm{BNNi}^{-}$fragments indicates that the synthesized $\mathrm{BN}$ is strongly coupled with the Ni substrate and hence justifies the asymmetric shape modeling adopted in photoemission spectra and the metallic character of the BN.

Figure 1a shows the N1s core level spectrum of a BN on Ni sample grown at $800^{\circ} \mathrm{C}$ for $2 \mathrm{~h}$. In this case, no $\pi$ plasmon signal is observed and the shape of the peak is asymmetric. On the other hand, after transfer of the BN film onto a Si(001) substrate, the $\pi$ plasmon signal was clearly detected (figure 1b), at $\sim 9$ eV from the main N1s peak [23-24]. The shape of the peak also changed to symmetric after transfer on $\mathrm{Si}$, as compared to the asymmetric shape found on the BN on Ni sample (see figures 1 for N1s and 2 for B1s). In addition, the boron and nitrogen peaks are shifted by $\sim 1 \mathrm{eV}$ after transfer (figures 1 and 2). Noticeably, the binding energy difference between the N1s and B1s peaks is identical before $\left(E_{N 1 s}-E_{B 1 s}=207.7 \mathrm{eV}\right)$ and after transfer $\left(E_{N 1 s}-E_{B 1 s}=207.6 \mathrm{eV}\right)$, a typical value for BN [10]. This suggests that the energy shift results from some Fermi level position change induced by the transfer from nickel to silicon.

The change in the shape of the B1s and N1s peaks from asymmetric to symmetric suggests that the non-detection of $\pi$ plasmons before transfer was due to the metallic nature of $B N$ induced by the strong interaction with the $\mathrm{Ni}$ substrate. Once this interaction is suppressed by transfer, the $\pi$ plasmons are detected, indicating the 2D nature of the h-BN layer. Thus, ToFSIMS and photoemission measurements independently confirm that the synthesized BN is strongly coupled with $\mathrm{Ni}$. If the crystallographic structure is similar to the h-BN one, its electronic structure is strongly affected by the bonding with the metallic Ni substrate. This analysis underlines the importance of the detection of $\pi$ plasmons to confirm the $2 D$ character of the synthetized material. 


\subsection{Spatial distribution of $h-B N$ :}

If the ToF-SIMS mapping (figure 4) shows a uniform presence of BN on the Ni surface, it does not give any information on its 2D character. The spatially-unresolved detection of $\pi$ plasmons after transfer indicates that at least some part of the BN bonds are h-BN. So, we have studied the spatial distribution of $\mathrm{h}-\mathrm{BN}$ by Raman and photoemission measurements with a micrometer probe size.

A peak associated to the intra-planar vibrations in $\mathrm{h}-\mathrm{BN}\left(\mathrm{E}_{2 \mathrm{~g}}\right.$ mode) should be detected by Raman spectroscopy toward $1370 \mathrm{~cm}^{-1}$ [31]. The detection of this peak, before and after transfer, was limited in our case to rarely detectable triangular domains with a characteristic dimension of $1 \mu \mathrm{m}$ (figure 6a). These domains are generally aligned, along grain boundaries, steps or other defects (see figure S4 in the supplementary material). This low intensity peak stands between 1368 and $1369 \mathrm{~cm}^{-1}$ with a full width at half maximum of $13-21 \mathrm{~cm}^{-1}$, identical before and after transfer (figure 6c). No BN Raman peak could be detected in areas indicated by black arrows. For example, no Raman peak is detected on a folded BN layer (figure 6b, black arrow in the upper left corner). This reflects the low BN Raman signal intensity at low thickness [32]. Thus, the non-detection of the Raman peak after transfer seems to result mainly from the low BN thickness. Nevertheless, wrinkles (marked by yellow arrows in figure 6a) are observed in these areas representing most of the surface. These wrinkles are not detected before growth, and result from the strain induced by the contraction of the $\mathrm{Ni}$ substrate during the cooling process [7, 33-34]. Their presence implies a full coverage of the surface by at least one BN layer.

Spatially-resolved photoemission measurements were carried out at the Antares beamline of the synchrotron SOLEIL. The N1s core-level mappings recorded before (figure 7a) and after BN transfer (figure $7 \mathrm{~b}$ ) indicate that nitrogen is detected everywhere on the surface. It should be noted that the minimal intensity (blue zones) do not correspond to a zero N1s signal. This distribution of intensity implies an inhomogeneous BN thickness, which may explain our previous observations predicting the presence of more than one monolayer of $B N(\sim 1.7 \mathrm{ML})$.

The thickness of BN in a multilayer area (denoted n.e for $n$ layers of thickness $e$ ) compared to the one in the thinnest domain (denoted $b$ because they correspond to the blue areas) was quantitatively determined by calculating their relative intensity ratio $I_{n . e} / I_{b}$ based on the photoemission attenuation equation [35]:

$$
I_{n, e}=I_{\infty}\left[1-e^{\left(-\frac{n_{i} \cdot \sin }{\lambda_{N_{B} B N} \sin \theta}\right)}\right]
$$

where $I_{n . e}$ represents the intensity measured for a $n$ layer thick BN film, $I_{\infty}$ the intensity of an infinite number of layers, $\lambda_{N, B N}$ the inelastic mean free path of an electron emitted by nitrogen in $\mathrm{BN}, \theta$ the angle of emission $\left(90^{\circ}\right)$ and $e$ the inter-planar distance in the h-BN (3.33 $\AA$ [36]). The $\lambda_{N, B N}$ value has been estimated to $6.8 \AA$ [37] for the photon energy of $700 \mathrm{eV}$. The calculation of the intensity ratio $I_{n . e} / I_{b}$ for the N1s core level is presented in table 1 . If it is assumed that the thickness of $B N$ in the thinnest zone $b$ is $2 \mathrm{ML}$ or more, the maximum calculated intensity ratio $I_{\max } / I_{b}$ between the intensities measured in the thickest and thinnest areas is 1.6. This value is inconsistent with the corresponding ratio deduced from the experimental mappings $\left(I_{\max } / I_{b}=1.8\right.$ and 1.9 respectively before and after transfer $)$. This 
shows that the $\mathrm{BN}$ thickness is $1 \mathrm{ML}$ for the thinnest $\mathrm{b}$ domains and $2 \mathrm{ML}$ for the domains which appear in green in figure 7. This distribution of the BN thickness is consistent with the non-spatially resolved photoemission measurements which lead to an average thickness of 1.7 ML in figure $3 a$.

The spatially-resolved photoemission spectra corresponding to nitrogen are presented in figure 8. Before transfer, $\pi$ plasmons are not detected (blue curve). After transfer, they are detected even for the lower intensity area (red curve). Although the detection is close to the noise signal, there is little doubt about their presence by comparison to the blue curve. The intensity of these $\pi$ plasmons increases for higher N1s intensities, thus for the thicker h-BN. All these results imply that 2D BN (h-BN) is present everywhere on the surface.

\section{Conclusion:}

By using borazine molecules, we synthesized a continuous BN film of large dimensions on polycrystalline $\mathrm{Ni}$ foils under UHV conditions in a MBE growth chamber. The BN thickness stands between 1 and $2 \mathrm{ML}$, a self-limitation that results from the catalytic effect of the nickel substrate. By combining ToF-SIMS with spatially and non-spatially resolved photoemission measurements, we confirm that a strong electronic coupling exists at the $\mathrm{h}-\mathrm{BN} / \mathrm{Ni}$ interface. The appearance of $\pi$ plasmons in photoemission spectra as well as the shape transformation of the XPS peaks from asymmetric to symmetric after transfer on silicon are two key results of this study, confirming the growth of h-BN. The detection of $\pi$ plasmons should be considered as a principal criterion validating the $2 \mathrm{D}$ nature of $\mathrm{BN}$.

Acknowledgments: We are indebted to Bérangère Toury (University of Lyon 1) for fruitful discussions concerning the borazine precursor. The financial supports by the 2DHetero FLAGERA project and the french RENATECH network are greatly acknowledged.

\section{References:}

[1] W. Paszkowicz, J.B. Pelka, M. Knapp, T. Szyszko \& S. Podsiadlo, Lattice parameters and anisotropic thermal expansion of hexagonal boron nitride in the 10-297.5 K temperature range, Appl. Phys. A, 75, 431 (2002).

[2] A. K. Geim \& I. V. Grigorieva, Van der Waals heterostructures, Nature, 499, 419 (2013).

[3] G. Cassabois, P. Valvin \& B. Gil, Hexagonal boron nitride is an indirect bandgap semiconductor, Nat. Photonics, 10, 262 (2016).

[4] L. Schué, L. Sponza, A. Plaud, H. Bensalah, K. Watanabe, T. Taniguchi, F. Ducastelle, A. Loiseau \& J. Barjon., Bright Luminescence from Indirect and Strongly Bound Excitons in h-BN, Phys. Rev. Lett., 122, 067401 (2019).

[5] C. R. Dean, A. F. Young, I. Meric, C. Lee, L. Wang, S. Sorgenfrei, K. Watanabe, T. Taniguchi, P. Kim, K. L. Shepard \& J. Hone, Boron nitride substrates for high-quality graphene electronics, Nat. Nanotechnol., 5, 722 (2010). 
[6] P. R. Kidambi, B. C. Bayer, R. Blume, Z.-J. Wang, C. Baehtz, R. S. Weatherup, M.-G. Willing, R. Schloegl \& S. Hofmann, Observing Graphene Grow: Catalyst-Graphene Interactions during Scalable Graphene Growth on Polycrystalline Copper, Nano Lett., 13, 4769 (2013).

[7] X. Li, W. Cai, J. An, S. Kim, J. Nah, D. Yang, R. Piner, A. Velamakanni, I. Jung, E. Tutuc, S. K. Banerjee, L. Colombo \& R. S. Ruoff, Large-Area Synthesis of High-Quality and Uniform Graphene Films on Copper Foils, Science, 324, 1312 (2009).

[8] A. B. Preobrajenski, S. A. Krasnikov, A. S. Vinogradov, M. L. Ng, T. Käämbre, A. A. Cafolla \& N. Mårtensson, Adsorption-induced gap states of h-BN on metal surfaces, Phys. Rev. B, 77, 085421 (2008).

[9] A. Nagashima, N. Tejima, Y. Gamou, T. Kawai \& C. Oshima, Electronic dispersion relations of monolayer hexagonal boron nitride formed on the Ni(111) surface, Phys. Rev. B, 51, 4606 (1995).

[10] A. B. Preobrajenski, A. S. Vinogradov \& N. Mårtensson, Ni 3d-BN $\pi$ hybridization at the hBN/Ni(111) interface observed with core-level spectroscopies, Phys. Rev. B, 70, 165404 (2004).

[11] M. Corso, W. Auwärter, M. Muntwiler, A. Tamai, T. Greber \& J. Osterwalder, Boron Nitride Nanomesh, Science, 303, 217 (2004).

[12] A.W. Laubengayer, P.C. Moews \& R.F. Porter, The condensation of borazine to polycyclic boron-nitrogen frameworks by pyrolytic dehydrogenation, J. Am. Chem. Soc., 83, 6 (1961).

[13] S. Mroz, C. Koziol \& J. Kolaczkiewicz, Carbon and sulphur on the [111] and [001] faces of nickel during thermal treatment in ultra-high vacuum and in an oxygen atmosphere, Vacuum, 26, 61 (1976).

[14] B. Dlubak, M-B. Martin, R. S. Weatherup, H. Yang, C. Deranlot, R. Blume, R. Schloegl, A. Fert, A. Anane, S. Hofmann, P. Seneor, \& J. Robertson, Graphene-passivated nickel as an oxidation-resistant electrode for spintronics, ACS Nano, 6, 10930, (2012).

[15] G. Deokar, J. Avila I. Razado-Colambo, J.-L. Codron, C. Boyaval, E. Galopin, M.-C. Asensio, \& D. Vignaud, Towards high quality CVD graphene growth and transfer, Carbon, 89, 82, (2015).

[16] S. Nakhaie, J. M. Wofford, T. Schumann, U. Jahn, M. Ramsteiner, M. Hanke, J. M. J. Lopes, \& H. Riechert, Synthesis of atomically thin hexagonal boron nitride films on nickel foils by molecular beam epitaxy, Appl. Phys. Lett., 106, 213108, (2015).

[17] H. Chou, A. Ismach, R. Ghosh, R. S. Ruoff \& A. Dolocan, Revealing the planar chemistry of two-dimensional heterostructures at the atomic level, Nature Comm., 6, 7482 (2015).

[18] Y. Uchida, S. Nakandakari, K. Kawahara, S. Yamasaki, M. Mitsuhara \& H. Ago, Controlled Growth of Large-Area Uniform Multilayer Hexagonal Boron Nitride as an Effective 2D Substrate, ACS Nano, 12, 6236 (2018).

[19] Y. Song, C. Zhang, B. Li, G. Ding, D. Jiang, H. Wang \& X. Xie, Van der Waals epitaxy and characterization of hexagonal boron nitride nanosheets on graphene, Nanoscale Res. Lett., 9, 367, (2014). 
[20] Y. Gao, Y. Zhang, P. Chen, Y. Li, M. Liu, T. Gao, D. Ma, Y. Chen, Z. Cheng, X. Qiu, W. Duan \& Z. Liu, Toward Single-Layer Uniform Hexagonal Boron Nitride-Graphene Patchworks with Zigzag Linking Edges, Nano Lett., 13, 3439 (2013).

[21] D. H. Berns \& M. A. Cappelli, Cubic boron nitride synthesis in low-density supersonic plasma flows, Appl. Phys. Lett., 68, 2711 (1996).

[22] R. Trehan, Y. Lifshitz \& J. W. Rabalais, Auger and x-ray electron spectroscopy studies of $h B N, c B N$, and $\mathrm{N}_{2}{ }^{+}$ion irradiation of boron and boron nitride, J. Vac. Sci. Technol. A, 8, 4026 (1990).

[23] F. Muench, M. Oezaslan, M. Rauber, S. Kaserer, A. Fuchs, E. Mankel, J. Brötz, P. Strasser, C. Roth \& W. Ensinger, Electroless synthesis of nanostructured nickel and nickel-boron tubes and their performance as unsupported ethanol electrooxidation catalysts, J. Power Sources, 222, 243 (2013).

[24] H. Li, H. Li, W.-L. Dai, W. Wang, Z. Fang \& J.-F. Deng, XPS studies on surface electronic characteristics of $\mathrm{Ni}-\mathrm{B}$ and $\mathrm{Ni}-\mathrm{P}$ amorphous alloy and its correlation to their catalytic properties, Appl. Surf. Sci., 152, 25 (1999).

[25] B. Feng, J. Zhang, Q. Zhong, W. Li, S. Li, H. Li, P. Cheng, S. Meng, L. Chen \& K. Wu, Experimental realization of two-dimensional boron sheets, Nature Chem., 8, 563 (2016).

[26] P. R. Kidambi, R. Blume, J. Kling, J. B. Wagner, C. Baehtz, R. S. Weatherup, R. Schloeg, B. C. Bayer \& S. Hofmann, In Situ Observations during Chemical Vapor Deposition of Hexagonal Boron Nitride on Polycrystalline Copper, Chem. Mater., 26, 6380 (2014).

[27] A.B. Preobrajenski, A. S. Vinogradov \& N. Mårtenssonet, Monolayer of h-BN chemisorbed on Cu(111) and Ni(111): The role of the transition metal 3d states, Surf. Sci., 582, 21 (2005).

[28] C. J. Powell et al., NIST Electron Effective-Attenuation-Length Database, Version 1.3, Standard Reference Data Program Database 82, National Institute of Standards and Technology, Gaithersburg, MD, (2011). https://www.nist.gov/srd/nist-standard-referencedatabase-82.

[29] S. Suzuki, R. Molto Pallares \& H. Hibino, Growth of atomically thin hexagonal boron nitride films by diffusion through a metal film and precipitation, J. Phys. D: Appl. Phys., 45, 385304 (2012).

[30] A. Yu, J. Lian, S. Siriponglert, H. Li, Y.P. Chen, S.S Pei, Graphene segregated on Ni surfaces and transferred to insulators, Appl. Phys. Lett., 93, 113103 (2008).

[31] S. Reich, A. C. Ferrari, R. Arenal, A. Loiseau, I. Bello \& J. Robertson, Resonant Raman scattering in cubic and hexagonal boron nitride, Phys. Rev. B, 71, 205201 (2005).

[32] R. V. Gorbachev, I. Riaz, R. R. Nair, R. Jalil, L. Britnell, B. D. Belle, E. W. Hill, K. S. Novoselov, K. Watanabe, T. Taniguchi, A. K. Geim \& P. Blake, Hunting for Monolayer Boron Nitride: Optical and Raman Signatures, Small, 7, 465 (2011).

[33] K. K. Kim, A. Hsu, X. Jia, S. M. Kim, Y. Shi, M. Hofmann, D. Nezich, J. F. Rodriguez-Nieva, M. Dresselhaus, T. Palacios \& J. Kong, Synthesis of Monolayer Hexagonal Boron Nitride on Cu Foil Using Chemical Vapor Deposition, Nano Lett., 12, 161 (2012). 
[34] J.-H. Park, J. C. Park, S. J. Yun, H. Kim, D. H. Luong, S. M. Kim, S. H. Choi, W. Yang, J. Kong, K. K. Kim \& Y. H. Lee, Large-Area Monolayer Hexagonal Boron Nitride on Pt Foil, ACS Nano, 8, 8520 (2014).

[35] M. P. Seah, Surface Analysis by Auger and X-Ray Photoelectron Spectroscopy, The Cromwell Press (Trowbridge), Chap. 13, Sec. 13.5, p. 369 (2003).

[36] M.I. Petrescu \& M.-G. Balint, Structure and properties modifications in boron nitride. Part I: Direct polymorphic transformations mechanisms, U.P.B. Sci. Bull., B, 69, 1454 (2007).

[37] J. Hadid, Croissance de BN hexagonal par épitaxie par jets moléculaires sur nickel, PhD thesis, University of Lille (2021). 


\section{Figure and table caption:}

Figure 1: N1s XPS core level spectra after growth for 2 hours at $800^{\circ} \mathrm{C}$ with $\mathrm{P}_{\text {borazine }}=3.5 \times 10^{-8}$ Torr, measured before $(a, c)$ and after transfer on Si (b, d). The $\pi$ plasmon peak is only detected after transfer (see inserts in panels $a$ and $b$ ). The corresponding best fits (blue curves) involve an asymmetric component in (c) and a symmetric one in (d). The black lines show the Shirley baseline.

Figure 2: B1s XPS core level spectra after growth for 2 hours at $800^{\circ} \mathrm{C}$ with $\mathrm{P}_{\text {borazine }}=3.5 \times 10^{-8}$ Torr, measured before (a) and after transfer on $\mathrm{Si}(\mathrm{b}, \mathrm{c})$. The corresponding best fits (blue curves) involve an asymmetric component in (a) and a symmetric one in (c). The Si plasmon contribution (b) was substracted in (c). The black lines show the Shirley baseline.

Figure 3: $d / \lambda_{B, B N}(\mathrm{a})$ and $\mathrm{B}: \mathrm{N}$ ratio (b) as a function of the growth temperature. All experiments were realized for a duration of 120 minutes. Dashed lines are guides, corresponding respectively to $d / \lambda_{B, B N}=0.17$ (a) and $\mathrm{B}: \mathrm{N}=1$ (b).

Figure 4: SEM Everhart-Thornley detector image for $\mathrm{BN}$ grown at $800^{\circ} \mathrm{C}$ for $2 \mathrm{~h}$ with $\mathrm{P}_{\text {borazine }}=$ $3.5 \times 10^{-8}$ Torr (a). ToF-SIMS maps of the same area as in a, showing respectively the total signal (b) and ${ }^{10} \mathrm{BN}^{-}$(c). The white circles in a and $\mathrm{b}$ show the correspondence between the SEM image and the ToF-SIMS cartography.

Figure 5: ToF-SIMS mass spectra of ${ }^{10} \mathrm{BN}^{-}$(a), $\mathrm{BNNi}^{-}, \mathrm{CHCl}_{2}^{-}$(b) and $\mathrm{Cl}^{-}(\mathrm{c})$, detected for $\mathrm{BN}$ grown at $800^{\circ} \mathrm{C}$ for $2 \mathrm{~h}$ with $\mathrm{P}_{\text {borazine }}=3.5 \times 10^{-8}$ Torr and on a Ni reference foil.

Figure 6: SEM images in InLens (a) and Everhart-Thornley mode (b) as well as the Raman spectra (c) detected on the domains marked by blue and black arrows in (a) and (b) for a sample transferred on a Si substrate. The yellow arrows in (a) indicate wrinkles appearing during the post-growth cooling step and are only observed in InLens mode.

Figure 7: N1s core level intensity mappings of BN on $\mathrm{Ni}$ foil (a) and after transfer on a Si substrate (b). The photon energy is $700 \mathrm{eV}$ and the integration time is $500 \mathrm{~ms}$ for (a) and 1000 ms for (b). (map dimensions: 100x80 $\mu \mathrm{m}^{2}$ ).

Figure 8: N1s photoemission spectra detected on the N1s cartography after transfer on Si (red and green curves), and before transfer (blue curve), vertically shifted for clarity. The blue and red curves correspond to a minimal N1s intensity and the green one to a maximal N1s intensity. The $\pi$ plasmons are only detected after transfer on the Si substrate.

Table 1: Calculated intensity ratio $I_{n . e} / I_{b}$ for the N1s peak as a function of BN thicknesses (2-10 $\mathrm{ML}$ for the multilayer domains and 1-3 ML for the $b$ domains). 
Figures and Table:

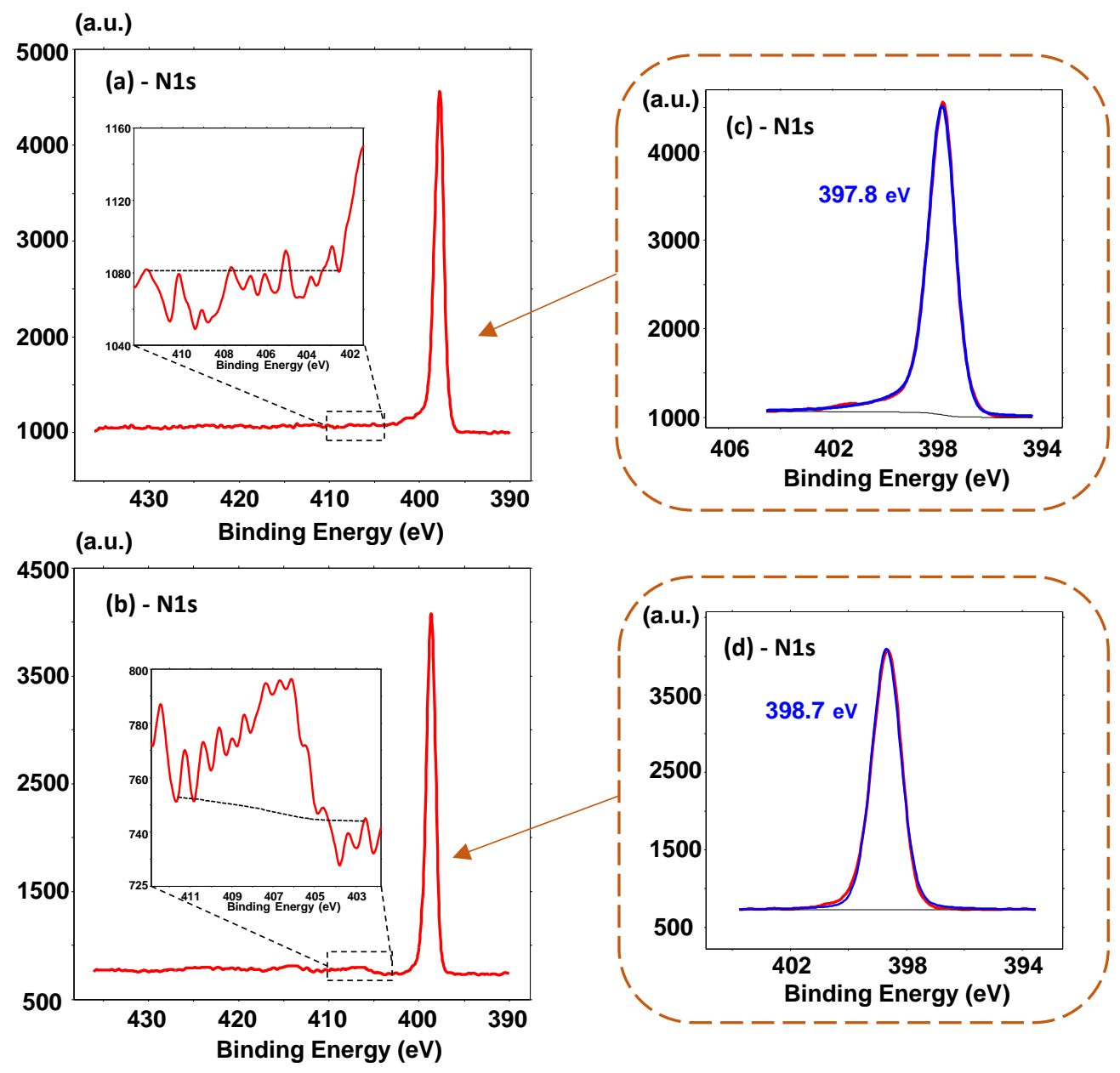

Figure 1
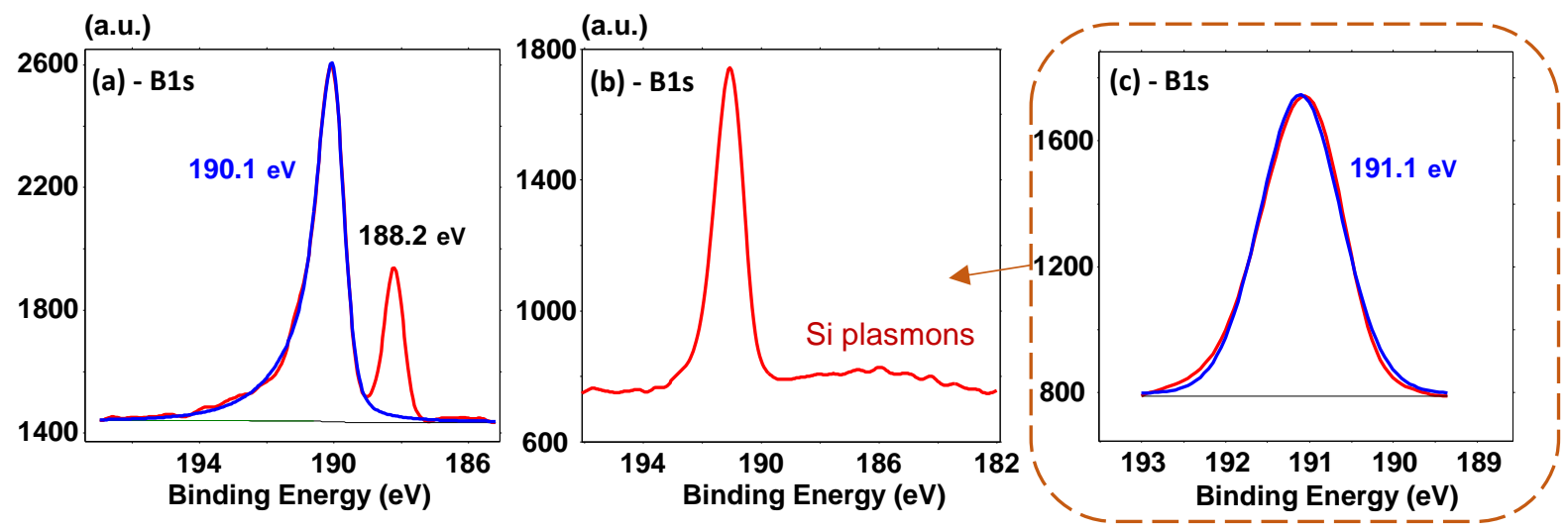

Figure 2 


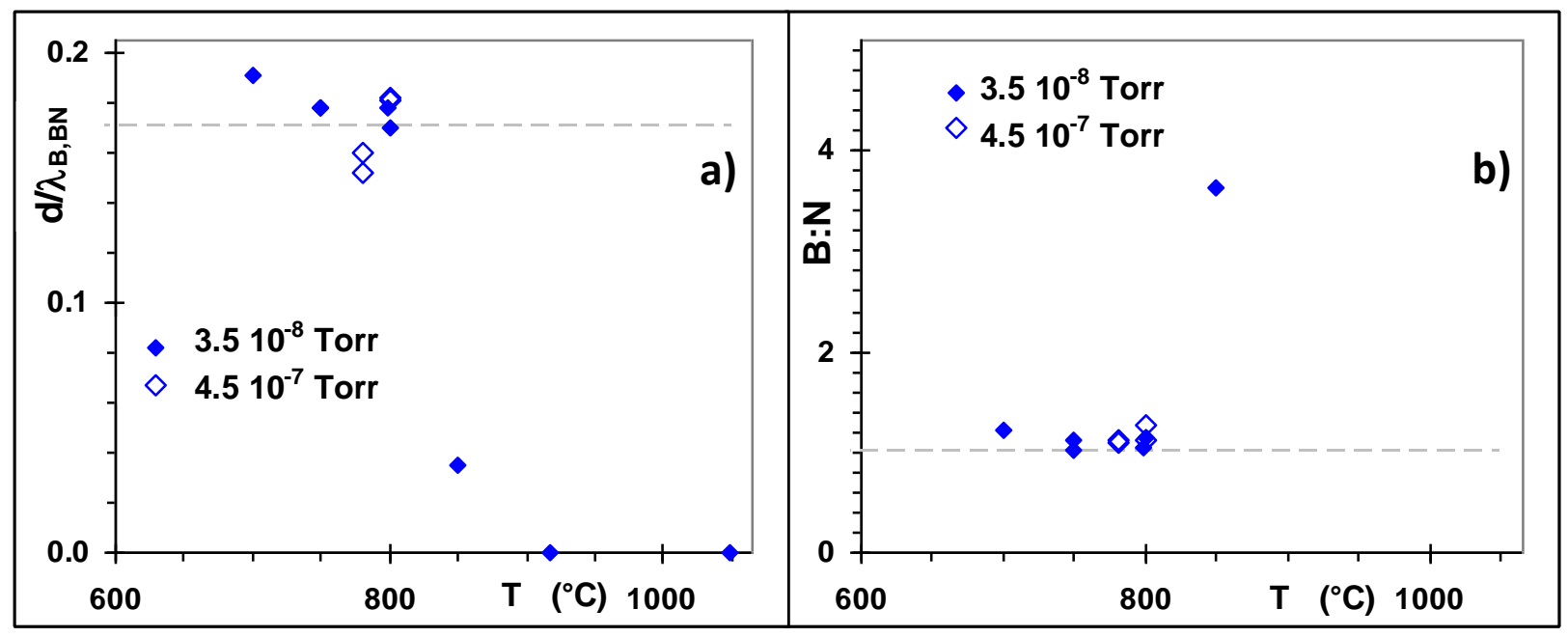

Figure 3
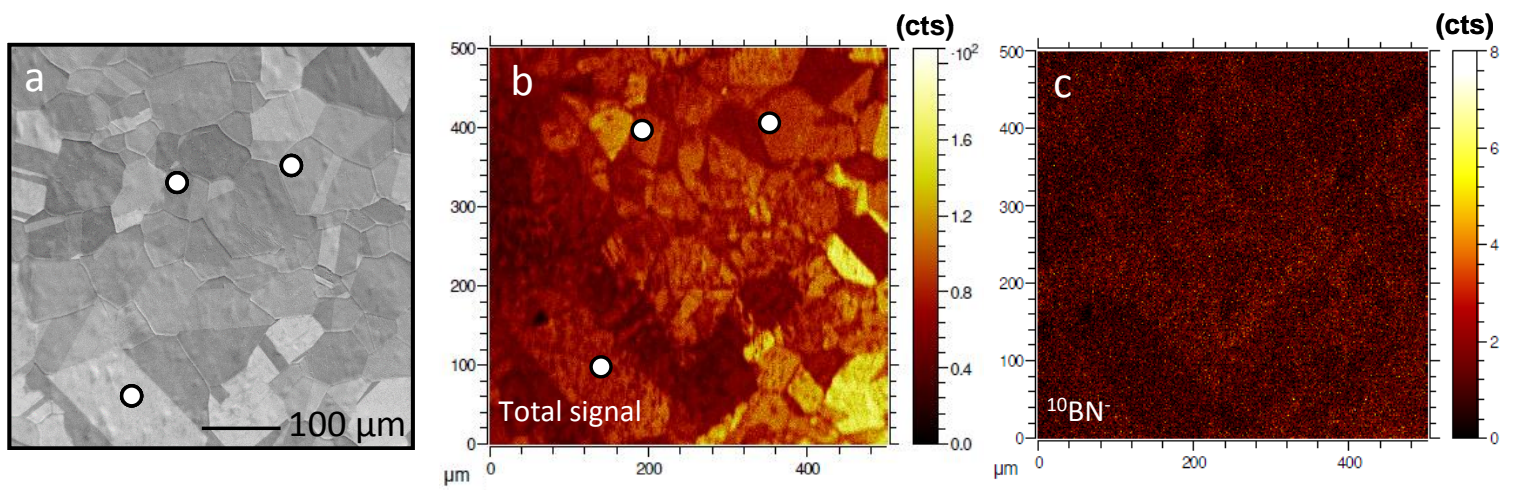

Figure 4 


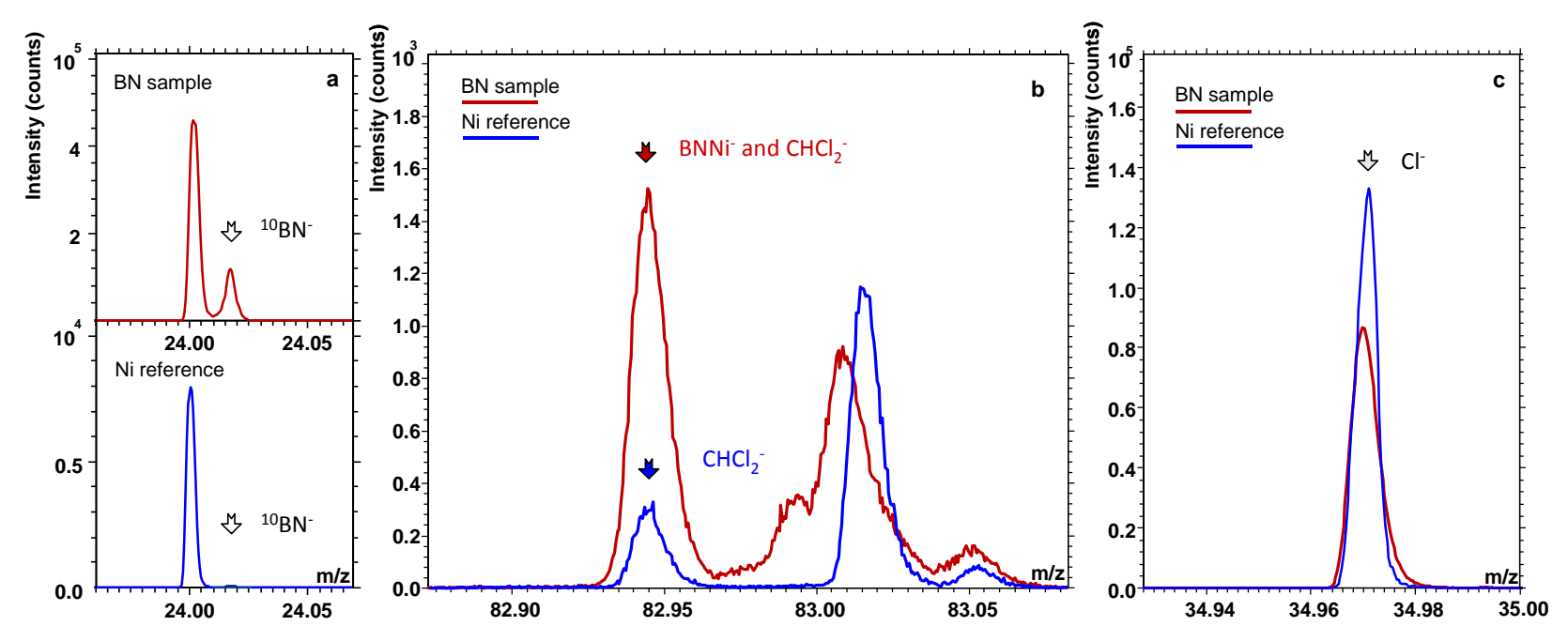

Figure 5
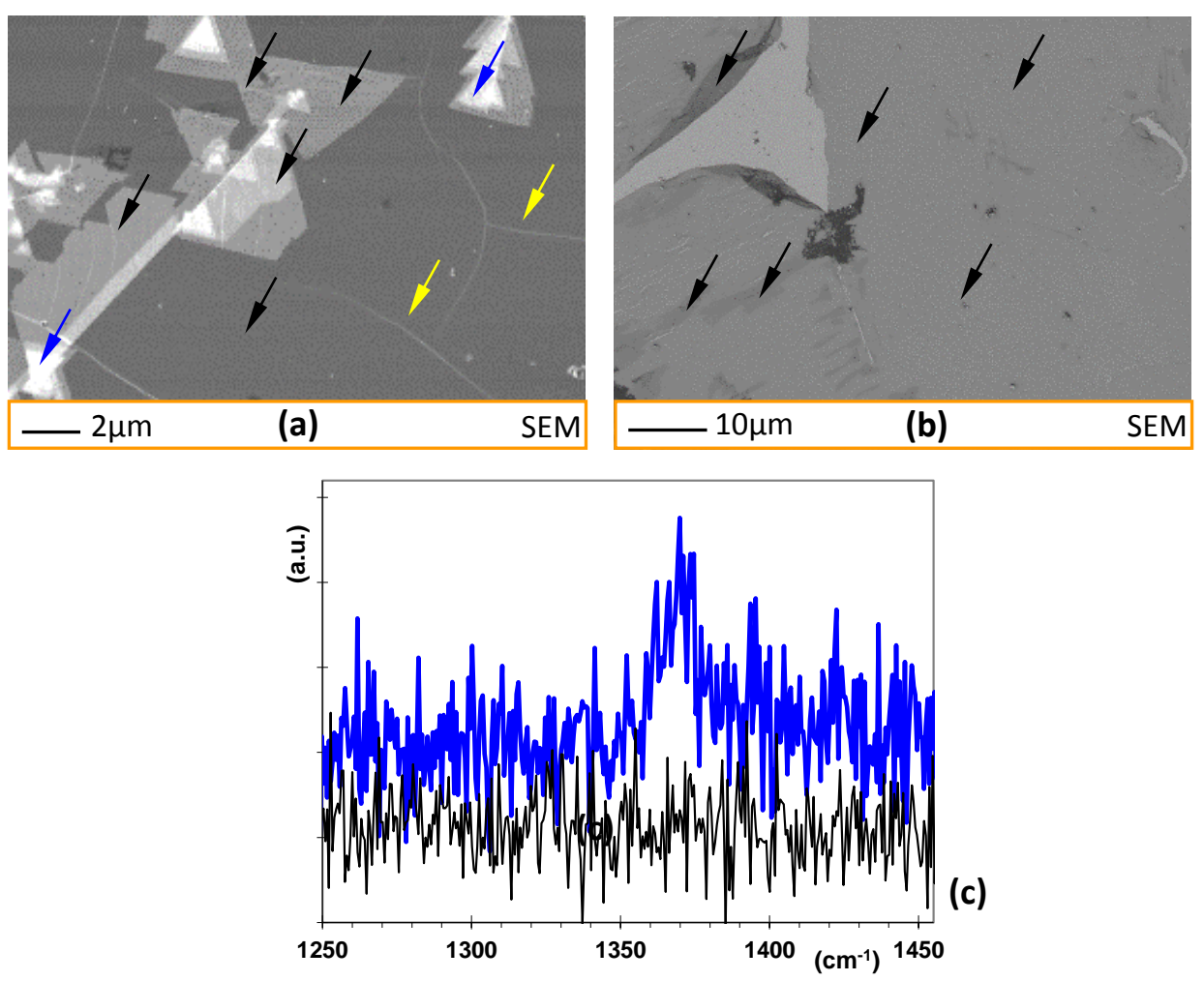

Figure 6 

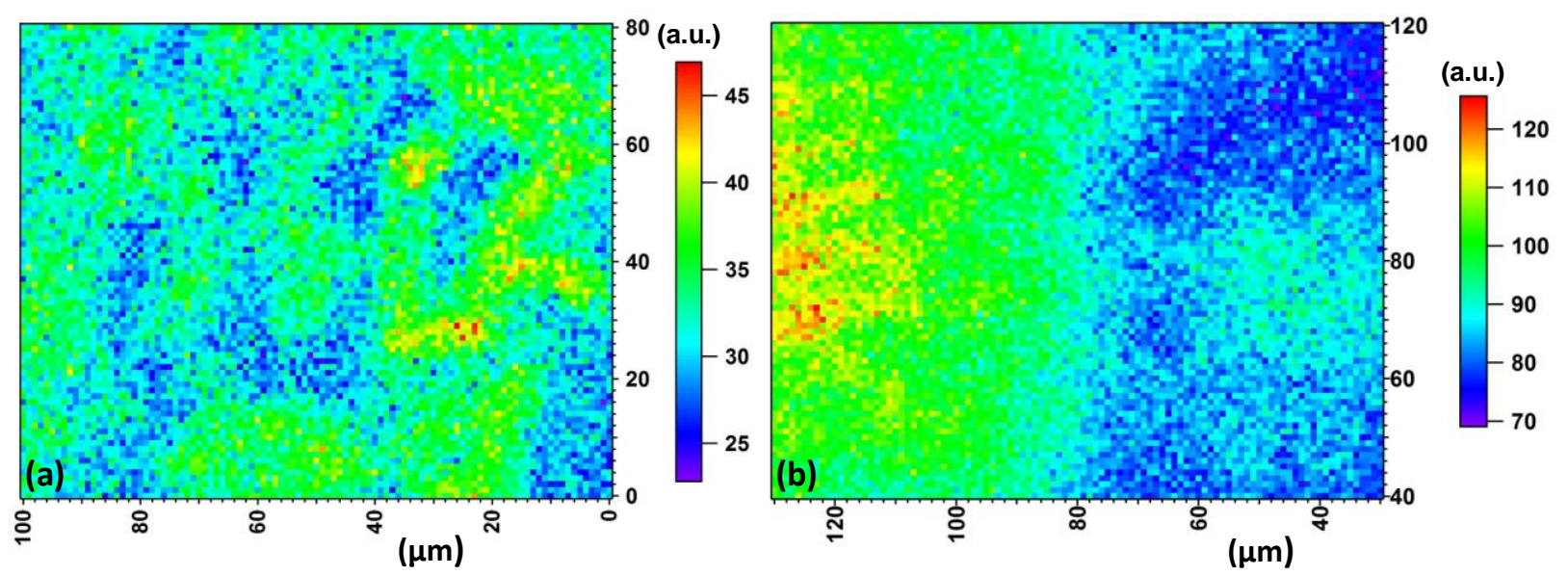

Figure 7

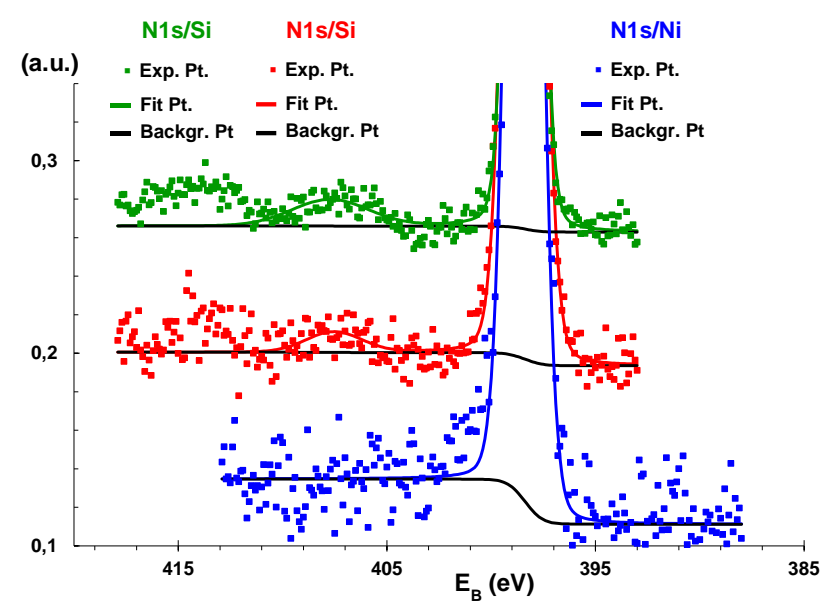

Figure 8

\begin{tabular}{cccc}
\hline n.e $b$ & $1 M L$ & $2 M L$ & $3 M L$ \\
\hline $2 M L$ & 1.61 & - & - \\
$3 M L$ & 1.99 & 1.23 & - \\
$\int$ & $\int$ & $\int$ & $\int$ \\
$11 M L$ & 2.57 & 1.59 & 1.29 \\
$12 M L$ & 2.58 & 1.6 & 1.3 \\
$13 M L$ & 2.58 & 1.6 & 1.3 \\
\hline
\end{tabular}

Table 1 